\title{
Cycling as a mode of transport for people over 30 years old on a mountainous town
}

\author{
Janaina Amorim Dias \\ Luiza Maciel Costa da Silva \\ Tectran, Systra Group, Brazil \\ Marconi Gomes da Silva \\ SPORTIF Clínica do Exercício e do Esporte, Brazil
}

\section{SUMMARY}

The relationship between active transportation and its role in health of population motivated the continuation of a pilot study evaluating the use of bicycles in a mountain town. The objective was to evaluate people over 30 years old, with different physical abilities, in a predefined route and check if the relief was impediment. The studied divided the riders into 2 groups: active and sedentary. It was performed a comparison of the physiological impact (heart rate, blood pressure) and a subjective perception (modified Borg's scale), ranking the degrees of difficulty during route stretches. The participants filled in a form before and after the course. Most sedentary participants, despite having greater difficulty and reaching higher heart rates on certain stretches, managed to make the trip. It suggests that the current technology of the bike allows people less physically conditioned to be able to use the bike in mountain cities.

\section{INTRODUCTION}

The world's largest cities are facing many problems regarding urban mobility and they seek solutions to this. Most developed countries have already advanced in this aspect, with large investments in projects, studies and implementation of more sustainable alternatives. On the other hand, most developing countries have even more investments focused in infrastructure for the use of cars to the detriment of quality, comfort and safety for pedestrians, cyclists and public transport.

From the 70s, with the growth of the automotive industry in Brazil, public transport policies have not benefited the transportation of masses through non-polluting means. In most large cities, there were no significant investments in infrastructure that would encourage alternative non-polluting transport, such as subway and bicycle. As a result, we can see an inefficient and chaotic system with long travel times, especially for poor people, as can be seen in major urban centers like São Paulo, Rio de Janeiro and Belo Horizonte, according to the National Public Transportation Agency- ANTP (2007).

The use of bicycles as a means of transport is being increasingly studied worldwide. Much 
of the work of recent years highlight the importance of bicycles as a mode of transport able to mitigate the problems of urban mobility and its impact on the health of the population from medium and large cities. However, few scientific work in developing countries such as Brazil, relate the importance of this vehicle in the urban displacement with the promotion of health. Most of the studies that make this correlation are from Europe and North America.

Importantly, the use of bicycles as a mode of transport as an isolated action does not solve the problems of congestion and pollution in large cities. The use of this non-motorized vehicle in the daily urban trips may have great importance for small and medium travel distances, especially when integrated with other modes of transport. Bike participation in the current scenario of large cities may represent a key element for the implementation of sustainable urban mobility, as a means of social inclusion, pollutants reduction and improvement of population health.

Physical inactivity is the fourth leading cause of mortality in the world due to chronic diseases - heart disease, stroke, diabetes and cancer - and every year these diseases result in more than three million deaths that could be prevented (World Health Organization, 2009). The latest global estimates indicate that $60 \%$ of the population is exposed to health risks due to physical inactivity. Increase physical activity is a priority in most countries (Bull FC TP Armstrong et al., 2005).

In order to keep up with changes in international recommendations of the World Health Organization - WHO (2010), since 2011 the practice physical activity indicator in leisure time is assessed regardless of a minimum number of days a week for physical activity. Thus, VIGITEL (Evaluation of risk and protective factors for chronic diseases through telephone survey, 2012) recommended at least 150 minutes per week of physical activity of moderate intensity or, at least 75 minutes a week of vigorous-intensity exercise.

In Brazil, the frequency of adults who practice the recommended level of physical activity during leisure time ranged from $27.9 \%$ in the city of São Paulo, to $43.1 \%$ in Florianopolis. Belo Horizonte showed $36.4 \%$ of its population as physically active (VIGITEL Brazil 2012).

Estimates of frequency and socio-demographic distribution of risk and protective factors for chronic diseases in capitals of 26 Brazilian states and the Federal District (VIGITEL, 2012) demonstrates that individuals who go to work or school by bike or walking (for at least part of the journey) and spend at least 30 minutes daily were considered physically active. The adults physically active displacements percentage varied between $10.5 \%$ in Palmas and $16.5 \%$ in Belém. Belo Horizonte presented $16.4 \%$ of its population as physically active based on displacement. Considering the entire adult population of the cities studied, adults physically active displacements were $14.2 \%$ in Brazil.

Based on this information, it aims a city model that is not only sustainable, but above all, a 
healthy city. Considering the importance of encouraging more sustainable mobility and the growing problems that a sedentary lifestyle has caused public health, it was developed a study involving bike use in Belo Horizonte, a mountainous town located in Brazil. The goal is to show that many sedentary people are able to use the bicycle as a mode of transport, including those who think that riding the hills of this city would only be possible with a very different physical capacity and that this mode of transport could only be booked for young people, free of any health problems.

Belo Horizonte has grown from 25,000 inhabitants to nearly 5 million people in its metropolitan area, in less than a century. People in this county did not create the displacement of culture with bicycles, possibly because at the beginning of bikes existence, they were all heavy and they didn't have gears, which greatly hindered its use in a city with mountainous terrain.

\section{OBJECTIVE AND METHODOLOGY}

The study objective was to apply an evaluation methodology for individuals with different functional capabilities (sedentary and physically active) in people over 30 years. The behavior was evaluated in a pre-established route in the city of Belo Horizonte, using the bicycle as a mode of transport. The study included 22 people, 11 physically active and 11 sedentary. The ages ranged between 30 and 59 years. People with serious heart or metabolic problems and high performance athletes were excluded. Qualifying people as active or sedentary followed the physical activity criteria recommended by the World Health Organization, which considered active those who made more than 150 minutes of exercise per week at moderate intensity or 75 minutes a week of vigorous physical exercise intensity.

All users have cycled the same route in similar and favorable weather conditions, with pleasant temperatures and no precipitation. Data were collected on weekends and holidays. The length of the route cycled was $5.2 \mathrm{~km}$, of which $3.8 \mathrm{~km}$ were in bike path and $1.4 \mathrm{~km}$ were on streets and sidewalks. All participants used the same bike type: Mountain Bike, Merida ${ }^{\circledR}$ brand, 27 speeds. Measurements of physiological parameters were performed (heart rate, blood pressure, symptoms presented) and the subjective perception of effort using the modified Borg scale (Fig. 1).

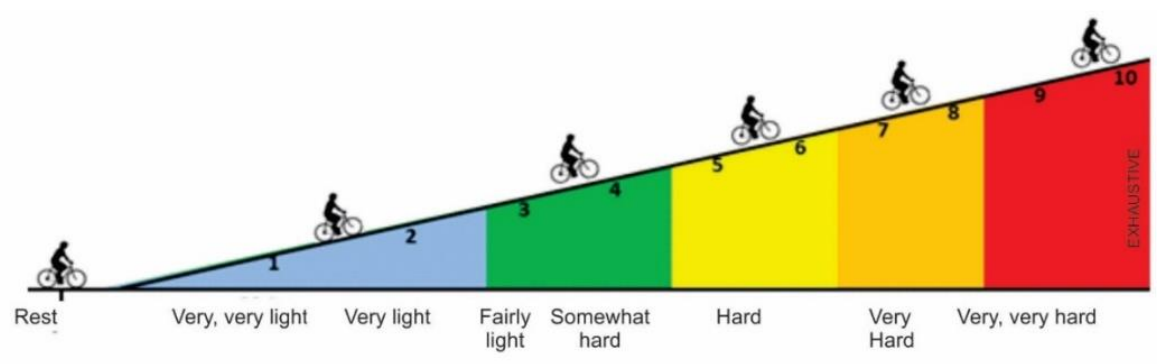

Fig. 1 - Modified Borg's scale 
Measurements were made individually and participants were oriented to follow their own pace, playing the shift to urban transportation purposes. The evaluated person was accompanied by a cyclist sport doctor and cardiologist who collected study data (heart rate and Borg scale). The doctor was responsible for interrupting the analysis in case of any complications with the participants. Heart rate was obtained with a heart rate monitor from Polar ${ }^{\circledR}$ brand (Model RS 400), so it wasn't necessary to stop cycling to obtain the data. Breaks on the route course were allowed to rest or if necessary (red light for cyclists). All cyclists carried a backpack $3 \mathrm{~kg}$ to simulate the average weight of an usual luggage.

A questionnaire were applied before and after the path, to collect anthropometric data, such as: age, height, weight, resting heart rate, body mass index (BMI), systolic and diastolic blood pressure and maximum heart rate estimated by age according to TANAKA formula, HR $\max =208$ - (age x 0.7). Participants also reported the time devoted to aerobic exercise per week, health problems known symptoms related to physical exercise, medication use, and lifestyle habits such as smoking, alcohol and drug use. They were also questioned about specific situations related to urban mobility, degree of satisfaction with the transportation and description of the advantages and disadvantages in the use of bicycles as a means of urban displacement.

Upon completion of the route, the participant estimated distance travelled and received the correct information. Also, participants were asked if that route was feasible in their everyday life and what are the main limiting factors for its implementation. The 1st and the 3rd measure had $8 \%$ declivity (Fig. 2).

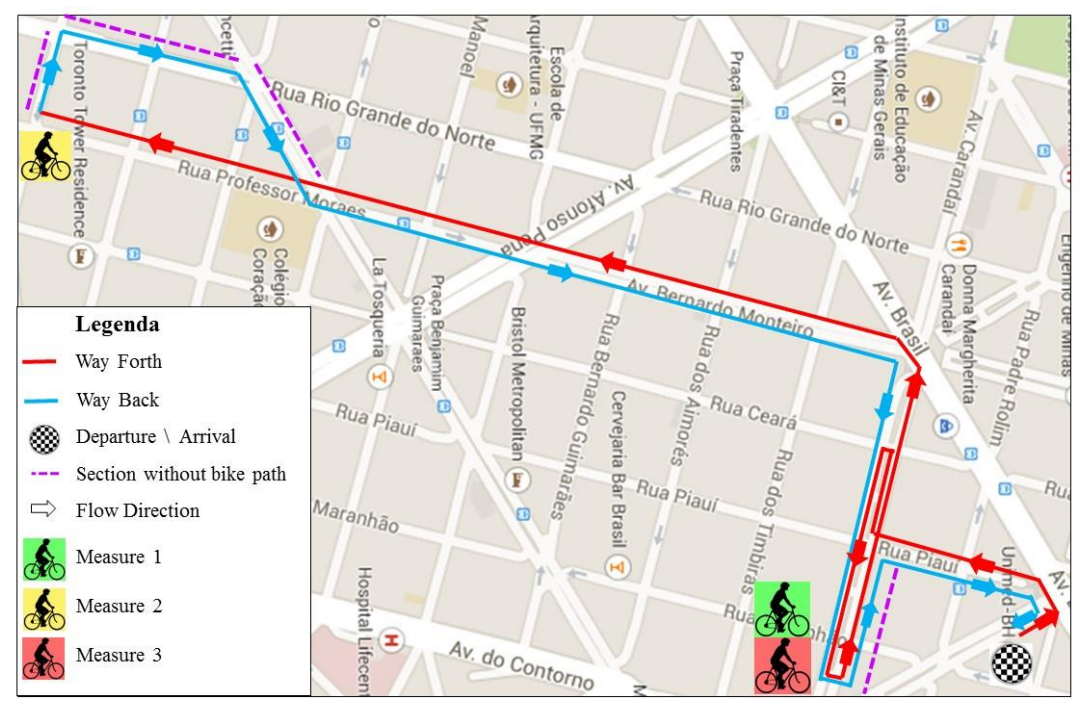

Fig. 2 - Coverage area of the route, central region of Belo Horizonte

It was an uphill route and cyclists rose about 63 meters during the ride. The database considered for this information was obtained in Prodabel. With this database it was developed analysis about the altimetry of the route using AutoCAD software, as can be seen in Fig. 3. 


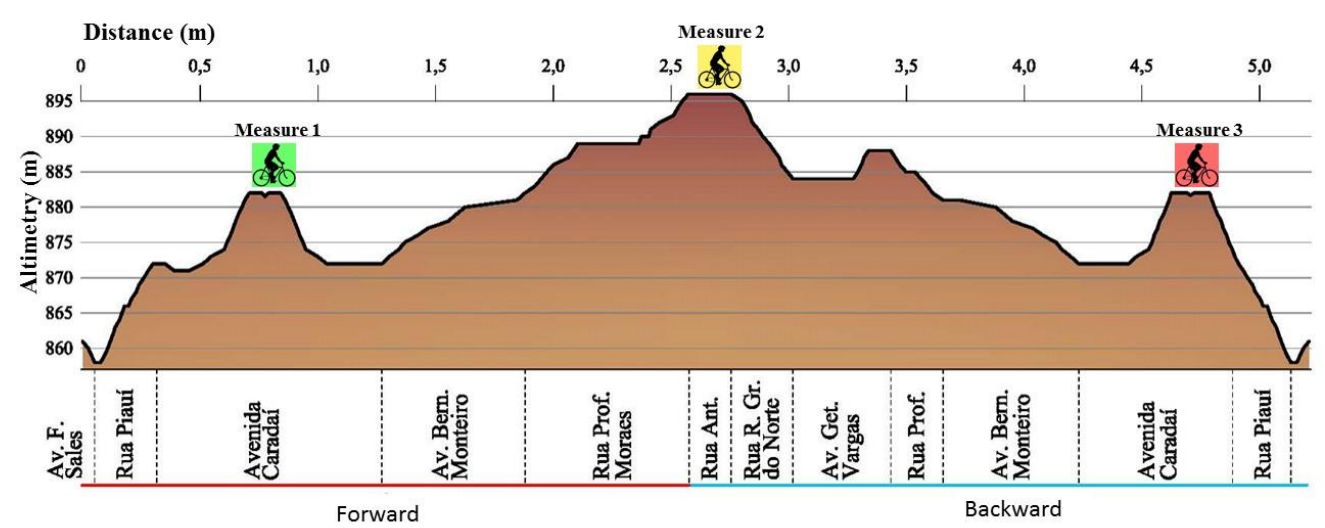

Fig. 3 - Altitude of the route

\section{ANALYSIS OF RESULTS}

\subsection{Statistical analysis}

The results were statistically analyzed using SPSS version 20.0 for Windows (SPSS Inc, Chicago, IL). All data were obtained with mean \pm standard deviation. The Mann-Whitney U test was used to compare the results between the 2 groups for statistical significance of $\mathrm{P} \leq$ 0.05 and the chi-square test or Fisher's exact test for categorical variables.

\subsection{Results}

22 people were analyzed, 11 active and 11 sedentary. In total group assessed, $48 \%$ reported the habit of using the bike, but none of them used it as a means of daily transport. Among the active cyclists, $44 \%$ said the altimetry of the land was a limiting factor. Among the sedentary cyclists, $91 \%$ felt altimetry of the land as an obstacle before cycling the defined route.

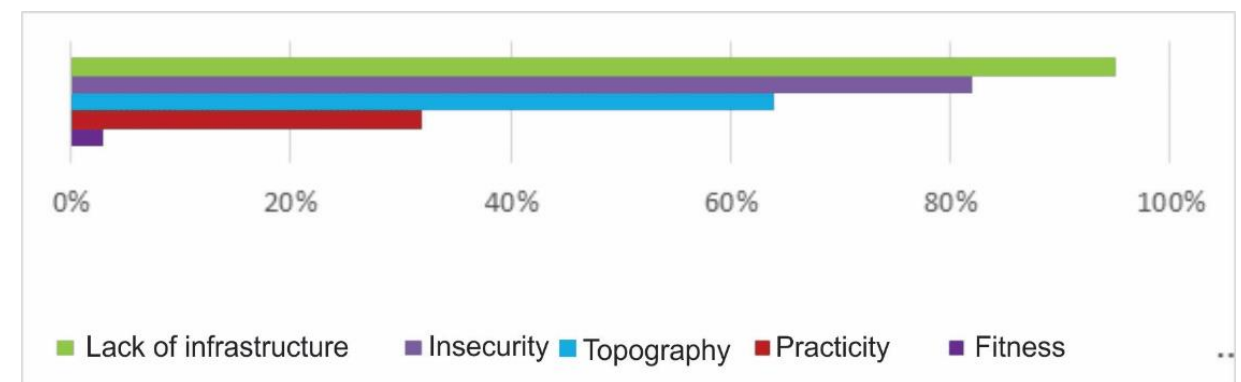

Fig. 4 - Factors that limit the use of bicycles in Belo Horizonte among participants

Three participants used drugs for high blood pressure and had blood pressure levels well controlled with, as can be seen in Table 1.

\begin{tabular}{lcccccc}
\hline \multicolumn{1}{c}{ Features } & \multicolumn{2}{c}{ Total (\%) } & \multicolumn{2}{c}{ Active } & \multicolumn{2}{c}{ Sedentary } \\
\hline Participants & 22 & $(100 \%)$ & 11 & $(50 \%)$ & 11 & $(50 \%)$ \\
\hline Hypertension & 3 & $(13,5 \%)$ & 2 & $(9 \%)$ & 1 & $(4,5 \%)$ \\
\hline Smokers & 1 & $(4,5 \%)$ & 0 & $(0 \%)$ & 1 & $(4,5 \%)$ \\
\hline Female & 10 & $(45 \%)$ & 5 & $(22,5 \%)$ & 6 & $(22,5 \%)$ \\
\hline Male & 12 & $(55 \%)$ & 6 & $(27,5 \%)$ & 6 & $(27,5 \%)$ \\
\hline
\end{tabular}

Table 1 - Group Features 
The average of time spend by cyclists to roam the stablished way was 26 minutes. People who were considered active were able to finish the route in na average of 6 minutes before of sedentary, as shown in Table 4:

\begin{tabular}{lccc}
\hline Characteristics & Total & Actives & Sedentary \\
\hline Average time of travel (min) & 26 & 23 & 29 \\
\hline $\begin{array}{l}\text { People who would do the route of 5,2 km as a } \\
\text { daily route }\end{array}$ & $18(82 \%)$ & $10(91 \%)$ & $8(73 \%)$ \\
\hline Felt safe during the travel & $19(86 \%)$ & $10(91 \%)$ & $9(82 \%)$ \\
\hline Average score ascent 1 - Borg scale & 3 & 2 & 3,5 \\
\hline Average score ascent 2 - Borg scale & 2 & 1,5 & 3 \\
\hline Average score ascent 2 - Borg scale & 3,5 & 3 & 4 \\
\hline Average score entire route - Borg scale & 3 & 2 & 4,5 \\
\hline Rest FC (\%) & 43 & 42 & 45 \\
\hline Ascent 1 average FC $(\%)$ & 82 & 80 & 85 \\
\hline Ascent 2 average FC $(\%)$ & 81 & 78 & 85 \\
\hline Ascent 3 average FC $(\%)$ & 88 & 85 & 91 \\
\hline Arrival average FC $(\%)$ & 80 & 77 & 84 \\
\hline
\end{tabular}

\section{Table 2 - Questionnaire concerning the route - Total group}

FC (\%) -percentage of heart rate in comparison with the maximum heart rate estimated for the participant's age by TANAKA's formula: Maximum FC estimated $=208-($ agex 0,7$)$

There was no heart beat variation during rest among groups. Heart beats measured on parts 1 and 3 were the highest ones in sedentary groups $(\mathrm{P}<0,05)$. Except for the ascent 2, no difference between sedentary's and active's heart beats was noticed.

In all route sections, the active group assigns Borg Scale scores lower than the sedentary group's scores $(\mathrm{P}<0.05)$. While the perceived exertion and heart rate in all sections have been greater in sedentary cyclists, most of them considered the route as one that recquires acceptable effort and classified as an almost hard intensity path (Bord Scale 4). Among physically active, most considered the path as easy (Borg Scale 2).

Table 7 shows data about the perception of participants in relation to the distance traveled. Most active and sedentary volunteers thought they had traveled less than $5.2 \mathrm{~km}$. Only one participant was able to correctly estimate the distance of the route (between $5 \mathrm{~km}$ and $6 \mathrm{~km}$ ). The most cited distance was within the range between $3 \mathrm{~km}$ and $4 \mathrm{~km}$ and any of them imagined to have traveled a longer distance than the one that was traveled.

How many kilometers do you think you have traveled on the route?

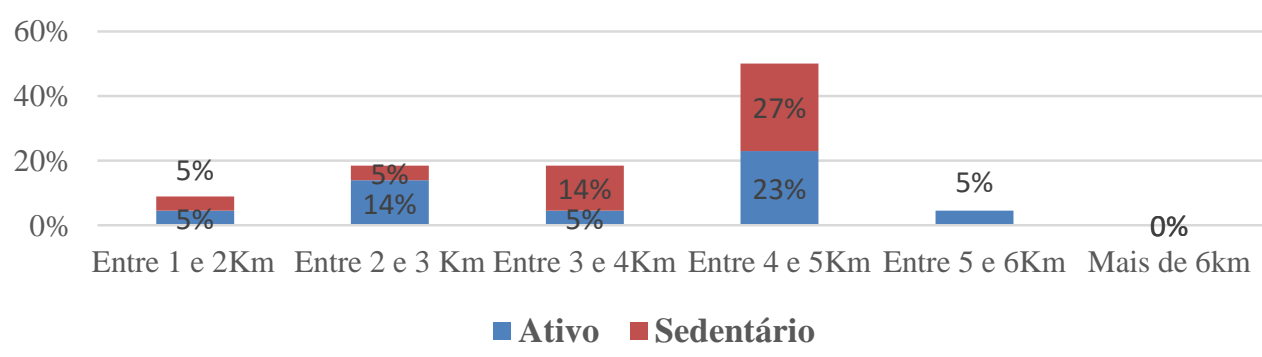

\section{Fig. 5 - Perception of the traveled distance}

$89 \%$ percent of the participants have said they would travel this route by bike, although only 
one volunteer currently used a bike as a meaning of transportation. $27 \%$ of the active participants classified their perception of riding a bike at Belo Horizonte as a lot easier than they imagined before, as shown at Fig. 8.

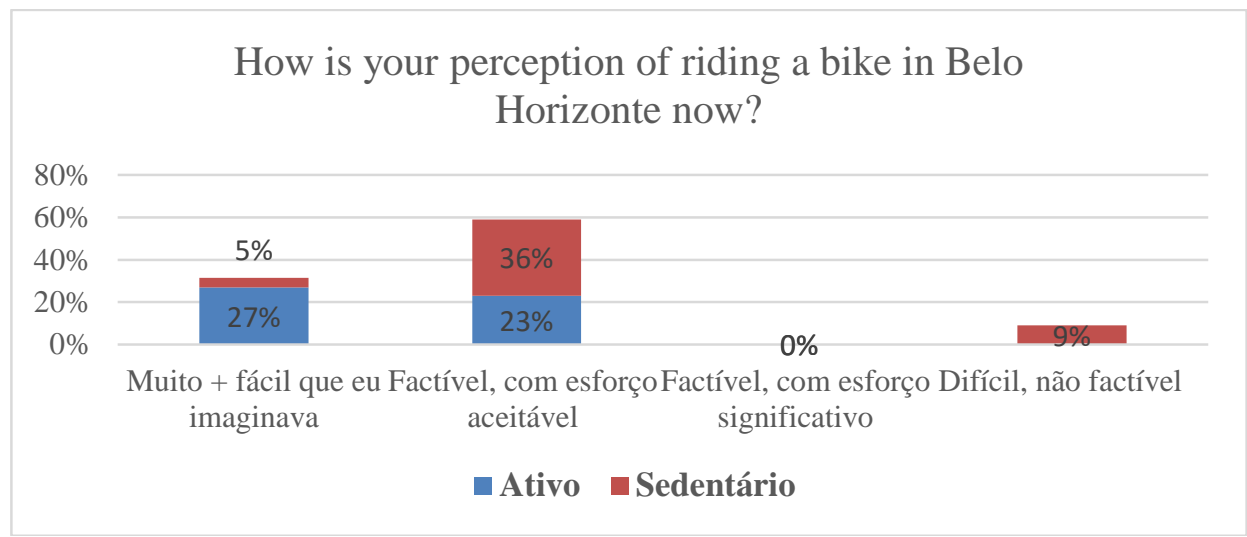

\section{Fig. 6 - Evaluation about cycling at Belo Horizonte}

\subsection{Analysis}

The idea of choosing a route almost entirely on bike path was to give greater security to participants. In addition, volunteers cycled during weekends and holidays, so the streets were calmer than regular days with heavy traffic of vehicles and pedestrians. Thus, it is assumed that the data on the perception of cycling in a big city may have been influenced by the facilities found during the weekends and holidays.

Interestingly, the scores assigned to the $3^{\text {rd }}$ measure were higher both in the sedentary group as in the group of actives, although the same increase in the $1^{\text {st }}$ measure. This may be explained by the physical stress that the participants presented in the course path. Thus, a subjective perception of greater effort on the last measure of the route was consistent.

It was found that the active participants made the journey on average 6 minutes faster than the sedentary group. The average heart rate of the active group was less than the sedentary in all route sections, with statistical significance in sections 1 and 3 . This result is consistent with the physiological cardiac, metabolic and musculoskeletal adaptations that physically active people acquire with conditioning physicist. From a physiological point of view, sedentary people needed a higher heart rate and reported a greater sense exertion by the modified Borg scale, just for not having these physiological adaptations that the human body develops exercise regularly.

Interestingly, although the perceived exertion and heart rate in all sections have been greater in sedentary cyclists, most of them thought the route as feasible with acceptable effort and classified as an intensity route between almost hard (Borg 4) and difficult (Borg 5). Among physically active, most considered the route as easy (Borg 2). These data show that sedentary people, within the proposed route could run it daily, becoming increasingly active people through active transport. With a better physical condition, these individuals have the perception of decreased effort and less increase in heart rate during the steepest climbs. This suggests that you can ride in Belo Horizonte and having a good physical condition only help to better complete the route in less time, less effort and more confidence.

The combination of physical inactivity, technical inability with the bike and the absence of a history of dedication to exercise, can be limiting factors in not only a mountainous town, 
but also anywhere. This corroborates with the guidelines of the World Health Organization recommends moderate or vigorous intensity exercise for health promotion.

\section{CONCLUSION}

The objection in cycling in a mountain town is not consistent with the reality of today, considering existing technology, where bicycles are increasingly lighter and have more features that make it easy to practice cycling with lower demand effort. Thus, most people with low physical capacities not felt unable to achieve the defined route in the study.

Given the difficulties that public health and urban mobility are facing, research and studies that encourage integrated projects are very important. Experts in public health, planning and urban transport as well as the World Health Organization data recognize the need to involve multi-sector policies for development projects in urban areas to encourage physical activity.

\section{REFERENCES}

\section{Book}

BULL FC, Armstrong TP et al. (2005) Comparative Quantification of Health Risks: Global and Regional Burden of Disease Attributable to Selected Major Risk Factors. Geneva: World Health Organization.

COMMISSION OF THE EUROPEAN COMMUNITIES (2005). Promoting Healthy Diets and Physical Activity: A European Dimension for the Prevention of Overweight, Obesity and Chronic Diseases.

LAFERRERE G. (2002) Comparison of national cycling policy in European Countries. Association for European Transport. Homerton College, Cambridge, England.

VIGITEL Brasil (2012) Vigilância de fatores de risco e proteção para doenças crônicas por inquérito telefônico: estimativas sobre frequência e distribuição sociodemográfica de fatores de risco e proteção para doenças crônicas nas capitais dos 26 estados brasileiros e no distrito federal em 2012.

WORLD HEALTH ORGANIZATION (2010) Global recommendations on physical activity for health. Geneva, Switzerland: World Health Organization.

WORLD HEALTH ORGANIZATION (2009) Global health risks: mortality and burden of disease attributable to selected major risks. Geneva, Switzerland: World Health Organization. 